\title{
DECIMAL/RESIDUE/DECIMAL OPTICAL CONVERTERS
}

\author{
David CASASENT and Demetri PSALTIS \\ Department of Electrical Engineering, Pittsburgh, PA 15213, USA
}

Received 12 June 1978

Revised manuscript received 21 August 1978

\begin{abstract}
An optical numerical processor using residue arithmetic is described. Specific designs and experimental demonstrations
\end{abstract} of decimal-to-residue and residue-to-decimal converters using a coherent optical processor are presented.

\section{Introduction}

Residue arithmetic [1-3] appears to be an attractive scheme for realizing a high speed numerical processor. Huang $[4,5]$ was one of the first to suggest fabrication of an optical residue arithmetic processor. One recent optical approach [6] to such a system uses the phase or polarization of a spatial point as the cyclic variable. Another approach [7] uses pulse-position modulation to represent numbers and achieves the necessary cyclic permutation by use of maps. Many possible realizations of optical residue arithmetic systems using integrated optical switches, planar waveguides, etc. have been suggested [7]. In this paper we address the design of a decimal/residue and residue/decimal converter. We present a correlation formulation of the required operations rather than expressing them by maps [7]. This approach leads directly to 2-D optical processor designs that can utilize the large available space bandwidth product of optical systems.

In residue notation, we represent a decimal number $X$ by its $N$ residues $R_{m_{i}}$ moduli $m_{i}$ (where $i=1$, $\ldots, N)$. All computations in residue arithmetic can thus be divided into sub-computations in each of $N$ moduli. Since there are no carries in residue addition, subtraction and multiplication, all subcomputations can be performed in parallel. Such systems are thus directly implementable in a parallel multichannel optical processor.

Furthermore, the maximum decimal number that can be represented in residue notation equals the product of the moduli. However, since a given computa- tion is divided into sub-computations in the $N$ moduli $m_{i}$, the dynamic range of each sub-computation is greatly reduced with an associated reduction in the accuracy required. Since the accuracy and dynamic range of an optical processor are often questioned, an optical residue arithmetic system is an attractive way to reduce the required dynamic range and improve the accuracy of the overall system. In residue arithmetic, this is achieved at an increased system space bandwidth (SBW). However, since the available SBW of an optical processor can rarely be fully utilized, this represents no limitation in practice. For these many reasons optical residue arithmetic systems have recently received considerable attention [4-7].

\section{Residue arithmetic}

For completeness, we briefly review residue arithmetic [1-3]. An integer $X$ is represented in a residue number system by the $N$-tuple set of residues $\left(R_{m_{1}}\right.$, $\ldots R_{m_{N}}$ ) with respect to $N$ different moduli $m_{i}$. The residues $R_{m_{i}}$ are the least positive integer remainders of the quotient of $X$ and $m_{i}$. To represent 13 in a residue system with moduli $(2,5,7,9)$, we divide 13 by the first modulus 2 and obtain 6 and a remainder or residue of 1 . We proceed similarly to divide 13 by the other moduli and find $13=(1,3,6,4)$. Similarly $59=$ $(1,4,3,5)$ in the same moduli.

To add these two numbers in residue, we add each residue number with respect to its respective modulus. We retain only the residue of the sum with respect to 
to the corresponding modulus. For example $13+59$ is

$m_{i}=(2,5,7,9)$

$13=(1,3,6,4)$

$59=(1,4,3,5)$

$72=(0,2,2,0)$

where for the far right residue number, $5+4=9$ or in modulo 9 this is 1 with a residue or remainder of 0 . The decimal sum $13+59=72$ is thus found to be $(0,2,2,0)$ in residue notation with moduli $(2,5,7,9)$.

To subtract two numbers in residue, we invert the subtrahend by complementing each residue number with respect to its modulus $m_{i}$. We then add these two numbers as before. To multiply two numbers in residue notation, we multiply each residue number and retain only the residue with respect to the corresponding modulus. As seen in all cases, no carries occur, each sub-computation is of vastly reduced dynamic range, and all sub-computations can be performed in parallel. Thus, the motivation for considering an optical residue arithmetic processor are apparent.

To perform computations optically in residue notation, we choose to denote decimal and residue numbers in pulse-position notation by the horizontal position of a pulse of light as Huang [4,5] and others [7] have chosen to do. Alternate representations involve denoting a number by the phase or polarization of a spot of light [6]. The conversion scheme we propose is based on a correlation formulation of residue arithmetic and is thus more directly realizable in other technologies besides coherent optics. We describe specific residue arithmetic architectures using coherent optical systems because such systems are readily realizable with present technology and because of the parallel processing and real-time high throughput of an optical processor.

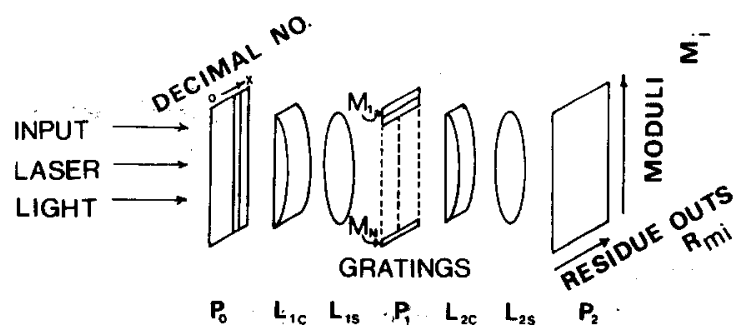

Fig. 1. Schematic diagram of a decimal-to-residue optical converter.

\section{Decimal-to-residue conversion}

We can represent any decimal integer $X$ by $X=n m_{i}$ $+R_{m_{i}}$, where $n$ is the number of times $X$ is divisible by $m_{i}$ and $R$ is the remainder or residue for the modulus $m_{i}$. Solving for the residue, we obtain $R_{m_{i}}=$ $X-n m_{i}$. To realize this conversion from decimal-toresidue, $m_{i}$ must be subtracted $n$ times from $X$ until the remainder satisfies $0 \leqslant R_{m_{i}} \leqslant m_{i}-1$. Multiplying these last two expressions by $\Delta x$ (unit distance in decimal and residue space) we obtain

$R_{m_{i}} \Delta x=\left(X-n m_{i}\right) \Delta x$,

$0 \leqslant R_{m_{i}} \Delta x \leqslant\left(m_{i}-1\right) \Delta x$.

Refer to the decimal-to-residue optical converter of fig. 1. Lenses $L_{1}$ and $L_{2}$ Fourier transform horizontally and image vertically. The input to this system (on one vertical channel) is the decimal number $X$ which, in pulse-position notation, is

$g\left(x_{0}\right)=\delta\left(x_{0}-X \Delta x\right)$.

We describe the system's impulse response for channel $i$ by

$h\left(x_{0}\right)=\sum_{n} \delta\left(x_{0}-n m_{i} \Delta x\right)$.

This is easily realized by a grating at $\mathbf{P}_{\mathbf{1}}$. The system's output is then

$$
\begin{aligned}
g \otimes h & =\sum_{n} \int \delta\left(x_{0}-X \Delta x\right) \delta\left(x_{0}-n m_{i}+x_{2}\right) \mathrm{d} x_{0} \\
& =\sum_{n} \delta\left[x_{2}-\left(X-n m_{i}\right) \Delta x\right] .
\end{aligned}
$$

From (5), we see that at $P_{2}$ of fig. 1 we find delta functions at $x_{2}=\left(X-n m_{i}\right) \Delta x$. The locations of these delta functions correspond to the various residues possible as described by (1). If we place a rectangular aperture properly centered at $\mathrm{P}_{2}$ of fig. 1 and of length $m_{i} \Delta x$, we can select the desired minimum positive $x_{2}$ value satisfying $0 \leqslant x_{2} \leqslant\left(m_{i}-1\right) \Delta x$, which is identical to (2).

Thus the output within this $\mathrm{P}_{2}$ aperture will consist of a peak of light whose location (in one of $m_{i}$ possible positions) within the aperture denotes the desired residue number $R_{m_{i}}$ modulo $m_{i}$. As depicted in fig. 1, to convert $X$ to $N$ residue numbers $R_{m_{i}}$ 
(where $N$ moduli $m_{i}$ are assumed), the input at $\mathrm{P}_{0}$ is a slit, $N$ gratings at frequencies $u_{g i}$ satisfying

$m_{i} \Delta x=u_{g i} \lambda f_{\mathrm{L}}$

are recorded on $N$ channels at $\mathrm{P}_{1}$, and $N$ channels appear at $\mathrm{P}_{2}$ with the position of the peak of light on each channel (within the $P_{2}$ aperture) denoting the residue of $X$ for the modulus $m_{i}$ of the particular channel.

\section{Experimental demonstration}

As the first experimental demonstration of optical residue arithmetic, we consider the conversion of the 21 decimal numbers 0 to 20 to residue with modulo 7. We represent the input $P_{0}$ in fig. 1 as shown in fig. $2 \mathrm{a}$ by 21 channels with a single pulse on each channel and an equal horizontal separation $\Delta x=0.7 \mathrm{~mm}$ be-

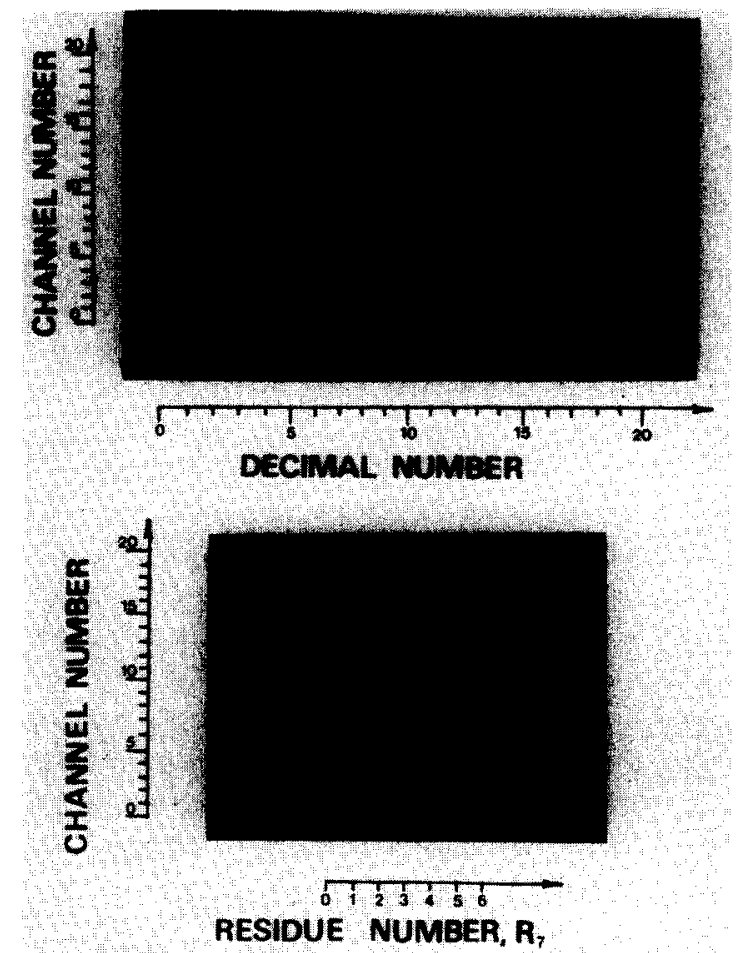

Fig. 2. Experimental demonstration of the decimal-to-residue conversion of the decimal numbers 0 to 21 to residue modulo 7 using the optical system of fig. 1 . (a) Input plane $P_{0}$ pattern, (b) Output plane $\mathrm{P}_{2}$ pattern. tween channels. This input pattern represents the decimal numbers 0 to 20 on the 21 channels from bottom to top in fig. $2 \mathrm{a}$ in pulse position coding. At $P_{1}$ of fig. 1 we place a grating of $10 \mathrm{cy} / \mathrm{mm}$ spatial frequency. With $\lambda=633 \mathrm{~nm}, f_{\mathrm{Lc}}=300 \mathrm{~mm}$ and $f_{\mathrm{Ls}}=762 \mathrm{~mm}$ focal length lenses, (6) is satisfied for a modulus $m_{i}=7$. The output $\mathrm{P}_{2}$ plane of fig. 1 is shown in fig. 2b. It contains 21 channels. The location of the output peak of light (in one of 7 possible locations) on each of the 21 channels represents the residue number $R_{m_{i}}$ modulo 7 for the corresponding decimal input number.

\section{Residue-to-decimal conversion}

The system of fig. 1 can also be used to perform residue-to-decimal conversion. In this case, the input $\mathrm{P}_{0}$ pattern consists of $N$ channels with a pulse of light on each channel at a horizontal location corresponding to the residue number $R_{m_{i}}$ with $R_{m_{i}}$ for different moduli recorded on different channels. At $\mathbf{P}_{1}$, we place $N$ gratings on $N$ lines with the grating frequencies corresponding to the $N$ moduli $m_{i}$ as in (6). At $\mathrm{P}_{2}$, we again find $N$ channels of delta functions. The horizontal locations of the delta functions on each channel now denote the various decimal numbers to which the input residue number could correspond. At one horizontal location in $\mathrm{P}_{2}$, all channels will have output peaks of light. This horizontal location denotes the decimal equivalent of the input set of residue numbers. The output detection is easily accomplished by an $N$ input NOR gate fed by a set of $N$ horizontally oriented linear self scanned photodiode CCD arrays.

\section{Discussion}

The pulse-position modulation scheme used requires an input and output space bandwidth of $M$ for the decimal/residue and residue/decimal converters respectively. With $M$ limited to 1000 for most practical spatial light modulators, the system will be severely limited. Several solutions to this problem exist. Firstly, the use of input data in binary rather than decimal representation would accomodate an input dynamic range of 64000 for 16 bits. Secondly, by raster recording the input data, $M$ values of 64000 could be accomodated by allowing 64 lines of 1000 points each 
to represent an input number. Thirdly, the decimal input can be used to control an oscillator's frequency which is raster recorded. In the 2-D Fourier transform of such a signal, a folded spectrum results with coarse and fine frequency axes. The location of the output peak along the fine frequency axis is the desired residue modulo the input horizontal line scan rate. In this case, a set of gratings is not required. Which of the approaches is best in the design of an optical residue arithmetic processor can best be determined by application to a specific problem.

\section{Acknowledgement}

The financial support of the National Science Foundation on Contract ENG 77-20038 for the work reported herein is gratefully acknowledged.

\section{References}

[1] H.L. Garner, The residue number system, IRE Trans. Electron. Comput., EC-8 (1959) 140.

[2] N.S. Szabo and R.I. Tanaka, Residue arithmetic and its applications to computer technology (New York, McGrawHill, 1967).

[3] A. Svoboda and M. Valach, in: Digital information processor, ed. W. Hoffman (John Wiley and Sons, Inc., NY, 1962).

[4] A. Huang, IOCC Conf., Washington, D.C., (April 1975) p. 14.

[5] A. Huang, Proc. EOSD, Anaheim, (Oct. 1977) p. 208.

[6] S. Collins, SPIE 128 (1977) 313.

[7] A. Huang, Y. Tsunoda and J.W. Goodman, Appl. Opt., submitted (1978). 